\title{
Evolution of Coronal Holes and Implications for High-Speed Solar Wind During the Minimum Between Cycles 23 and 24
}

\author{
G. de Toma
}

Received: 16 September 2010 / Accepted: 6 November 2010 / Published online: 30 November 2010

(C) Springer Science+Business Media B.V. 2010

\begin{abstract}
We analyze coronal holes present on the Sun during the extended minimum between Cycles 23 and 24, study their evolution, examine the consequences for the solar wind speed near the Earth, and compare it with the previous minimum in 1996. We identify coronal holes and determine their size and location using a combination of EUV observations from SOHO/EIT and STEREO/EUVI and magnetograms. We find that the long period of low solar activity from 2006 to 2009 was characterized by weak polar magnetic fields and polar coronal holes smaller than observed during the previous minimum. We also find that large, low-latitude coronal holes were present on the Sun until 2008 and remained important sources of recurrent high-speed solar wind streams. By the end of 2008, these low-latitude coronal holes started to close down, and finally disappeared in 2009, while smaller, midlatitude coronal holes formed in the remnants of Cycle 24 active regions shifting the sources of the solar wind at the Earth to higher latitudes.
\end{abstract}

Keywords Solar cycle $\cdot$ Coronal holes $\cdot$ Solar wind speed

\section{Introduction}

The slow decline of solar Cycle 23 and slow rise of Cycle 24 resulted in a very long period of low solar activity which lasted from about 2006 to the end of 2009, with 2008 and 2009 being particularly quiet years. This long, quiet period was characterized by limited magnetic flux emergence at the photosphere, mostly in the southern hemisphere, and low CME and flare activity in the corona. Figure 1 shows the sunspot record going back to 1850 with each spotless day marked by a black line at the bottom. Table 1 gives the mean sunspot number for the previous minima in comparison with the years 2006-2009. These records clearly show that the minimum between Cycles 23 and Cycle 24 was a very long and deep

The Sun - Earth Connection near Solar Minimum Guest Editors: M.M. Bisi, B.A. Emery, and B.J. Thompson

G. de Toma $(\bowtie)$

NCAR/HAO, 3080 Center Green, Boulder, CO, USA

e-mail: detoma@ucar.edu 


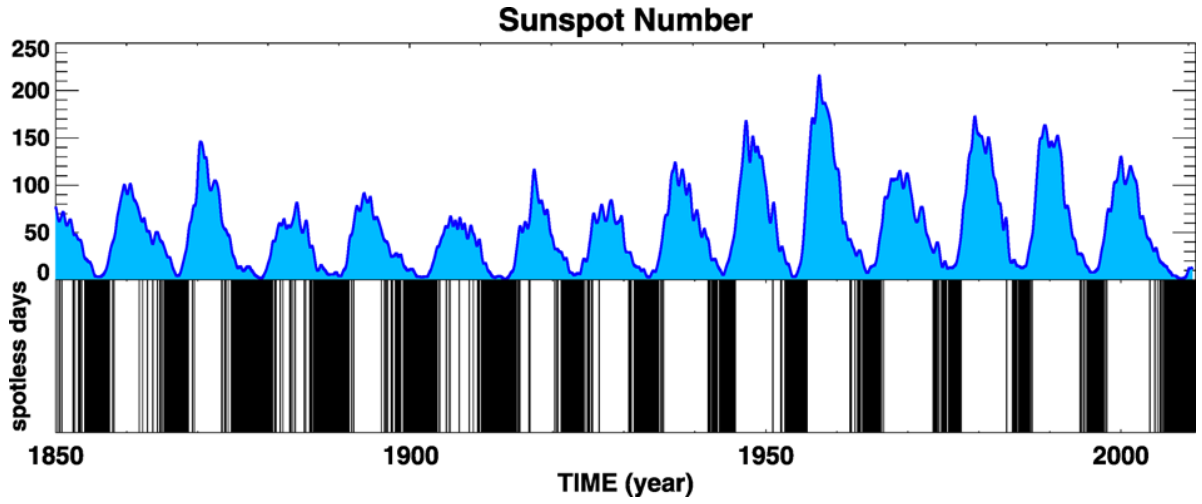

Figure 1 Time series of mean sunspot number averaged using a running six-rotation Gaussian function from 1850 to June 2010 (top). Days with no spots are identified by black bars at the bottom. This minimum was longer and quieter than other recent minima, however, long and very quiet minima were common at the beginning of the 1900 s and in late 1800 s.

Table 1 Yearly Mean Sunspot Number for the solar minima in 1976, 1986, and 1996 and for the years 2006-2009. In 2007 sunspot activity was already below the level observed during the previous minima and dropped to extremely low levels in 2008 and 2009.

\begin{tabular}{llllllll}
\hline Year & 1976 & 1986 & 1996 & 2006 & 2007 & 2008 & 2009 \\
Sunspot number & 12.6 & 13.4 & 8.6 & 15.2 & 7.6 & 2.9 & 3.1 \\
\hline
\end{tabular}

solar minimum. Already in 2006, the Sun showed a sunspot activity level similar to what was observed during the previous minima and activity continued to steadily decline in the following years. In 2008-2009 sunspot activity reached the lowest level observed since 1913. Both in 2008 and 2009 there were over 260 days without spots, i.e. spots were present on the Sun less than $30 \%$ of the time. We need to go back about a century to the $1911-1913$ or 1901-1902 minima to find solar cycles that had such a deep and long solar minimum.

An interesting aspect of the minimum between Cycles 23 and 24 was that the polar magnetic fields at the photosphere were weaker than in 1996 and remained weak throughout the minimum phase (see, e.g., Sheeley, 2008; de Toma and Arge, 2010; Wang, Robbrecht, and Sheeley, 2009). Figure 2 shows the net magnetic flux near the poles between $60^{\circ}$ and $80^{\circ}$ latitude for the northern and southern hemispheres observed by SOHO/MDI and at NSO/Kitt Peak. The net magnetic flux was relatively constant from 2005 to 2009 and about half of that observed in 1996, and by 2010 started to slowly decline. These lower flux values were in part due to a lower magnetic flux density near the poles and in part because of a more mixed polarity at high latitudes than in 1996 (de Toma and Arge, in preparation). Polar magnetic fields are known to play a very important role in shaping the solar corona and heliosphere at or near solar minimum when the polar dipole moment is the dominant component of the large scale magnetic field of the Sun (see, e.g., Wang and Sheeley, 2002). Thus, this different magnetic configuration gave rise to a different morphology of the corona and heliosphere from the ones observed in 1986 and 1996, when polar magnetic fields were stronger. During the years 2006-2009 the polar dipole and octupole moments were smaller than in 1996, while other moments with $m$ not equal to zero did not decrease much compared to 1996. This resulted in an overall smaller ratio between the polar dipole and higher order moments (Abramenko et al., 2010; 


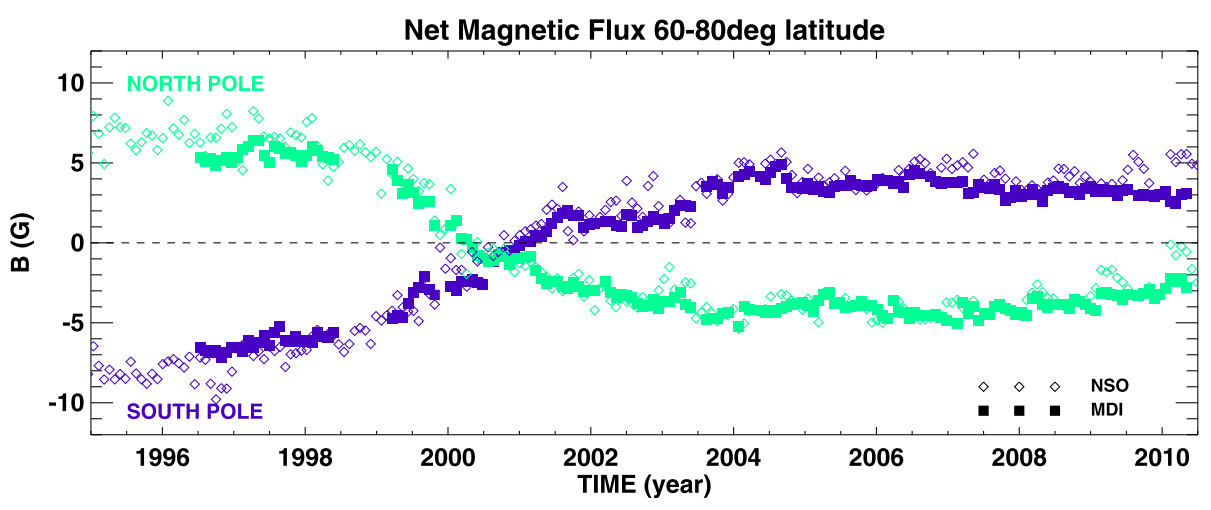

Figure 2 Net magnetic flux in the polar regions between $60^{\circ}$ and $80^{\circ}$ latitudes as measured by MDI on SOHO and at the National Solar Observatory (NSO) at Kitt Peak.

de Toma and Arge, 2010) which allowed the corona and heliosphere to retain some complexity from the late declining phase of solar Cycle 23 into the solar minimum phase (see, e.g., McComas et al., 2006, 2008; Gibson et al., 2009; Lee et al., 2009; Luhmann et al., 2009; de Toma et al., 2010a, 2010b). Consequently, during the minimum between Cycles 23 and 24 , the solar corona never reached a simple dipolar configuration with coronal streamers confined within a narrow latitude band near the equator. Instead, multiple streamers extending to relatively high latitudes were still present in 2008 and 2009 (de Toma et al., $2010 \mathrm{~b}$ ). Even the outer corona and heliosphere remained more complex than observed in 1996 for a significant fraction of this long minimum (see, e.g., Tokumaru et al., 2009; Tokumaru, Kojima, and Fujiki, 2010). In spite of the record low magnetic fields observed in the heliosphere (Smith and Balogh, 2008), the heliospheric current sheet was still relatively warped in 2007 and 2008 and started to flatten only in 2009 (de Toma et al., 2010a).

Coronal holes also differed during this minimum compared to the previous one, as we will discuss in more detail in Section 2. When the Sun reached the minimum activity between Cycle 22 and Cycle 23 in 1996, low-latitude coronal holes - that are commonly seen during the declining phase of the solar cycle - were almost completely absent from the Sun. In 2007 and 2008, in spite of the extremely low sunspot activity, coronal holes at low-latitudes located in weak, old-cycle active region remnants were still relatively large. These low-latitude coronal holes remained important sources of the solar wind at the Earth until the end of 2008, producing fast and recurrent solar wind streams with clear 9-, 13.5- and 27-day periodicities (Emery et al., 2009; Gibson et al., 2009; Lei et al., 2010). In 2009, with the decay of Cycle 23 active complexes, the low-latitude coronal holes disappeared, so did the high-speed streams in the solar wind. At about the same time, as Cycle 24 activity increased, small and short-lived coronal holes formed at mid latitudes at the locations where new-cycle active regions had emerged.

While the minimum between Cycles 23 and 24 was not unusual in a historical perspective, it differed in many ways from other recent minima. As pointed out by Gibson et al. (2011), this was the first time that the Sun was observed in such a long and quiet state with a large array of instruments from both ground and space observatories. It, thus, offered a unique opportunity to learn about the Sun and the Sun-Earth interaction during extended minima. The Whole Heliosphere Interval (WHI) observing campaign ran from 20 March to 16 April 2008, which corresponds to Carrington rotation (CR) 2068, and was part of an international effort to observe the Sun during its minimum state (Gibson et al., 2011, 
this issue). Because magnetic activity continued to decline in 2008 and 2009 and because the corona and heliosphere continued to evolve throughout this extended minimum, two additional periods were added to the original WHI rotation to better characterize this long minimum: WHI2 from 17 Dec 2008 to 12 January 2009 (corresponding to CR 2078) and WHI3 from 26 June to 22 Jul 2009 (corresponding to CR 2085). The original WHI rotation in spring 2008 well represents the coronal hole configuration seen at the Sun in 2007 and 2008, when large, low-latitude coronal holes were present. The other two WHI periods happened at times when these low-latitude coronal holes started to close down (WHI2) and when they had disappeared and smaller, shorter-lived, mid-latitude holes started to form in decayed, Cycle 24 active regions (WHI3). Hence, these three periods together well illustrate the coronal hole evolution during this long solar minimum.

In this paper we investigate the coronal hole changes during the years 2007-2009 and their effect on the solar wind speed near the Earth with special emphasis on the three WHI periods. In Section 2, we use an automatic detection scheme to determine the size and location of coronal holes during the past two minima. We give estimates of the coronal hole area and study the evolution of coronal holes during the two minima. In Section 3, we examine how the solar wind speed distribution changed in response to the coronal hole changes at the Sun. Conclusions are given in Section 4. The technique used to identify coronal holes from solar EUV images is described in the Appendix.

\section{Coronal Holes}

Observationally, coronal holes are typically defined as regions of low emission in the solar corona because they appear as relatively dark regions in X-rays and EUV. Because of the strong association between coronal holes and high-speed solar wind streams which has been known since the 1970s (see, e.g., Krieger, Timothy, and Roelof, 1973; Neupert and Pizzo, 1974; Nolte et al., 1976; Zirker, 1977), and because of the detection of blue shift within coronal holes in coronal and transition region lines indicating outflow (see, e.g., Hassler et al., 1999; Peter and Judge, 1999; Xia, Marsch, and Curdt, 2003; Aiouaz, Peter, and Lamaire, 2005) coronal holes are usually identified as the sources of the fast wind from where the wind flows out in the corona and is accelerated in expanding magnetic funnels (Tu et al., 2005). However, in coronal models, the term coronal holes is often used to more generally indicate the foot-points of the magnetic field lines "open" to the heliosphere. The two definitions are not equivalent, and not all magnetically open field lines must be necessarily rooted within the observed coronal holes. In this paper, we use the term coronal holes to define regions of one dominant magnetic polarity and reduced EUV emission in the corona and assume they are source regions at the Sun of fast solar wind (see the recent review of Cranmer (2009) and the references therein for a more complete discussion of coronal holes and the origin of solar wind).

Coronal holes are not easy to identify in solar observations. They have lower electron density and temperature than the typical quiet Sun; thus they appear as dark regions in EUV and X-ray images (see, e.g., Zirker, 1977). However, intensity does not uniquely distinguish coronal holes from other regions on the Sun. For example, filament channels and some quiet Sun regions can also appear as dark as coronal holes (de Toma and Arge, 2005). Moreover, in optically thin coronal lines, bright structures intercepting the line-of-sight can partially obscure the boundaries of coronal holes. Therefore, the identification of coronal holes using intensity images is often ambiguous, as they appear slightly different in shape and area at different wavelengths (see, e.g., Kahler, Davis, and Harvey, 1983). Ideally, detection of blue-shifted regions would be a better means to identify coronal holes, but velocity 
estimates in the corona and chromosphere are difficult and not routinely available, so it is customary to use intensity images to detect coronal holes. Historically, the best determinations of coronal holes were drawings made by careful and experienced observers (see, e.g., Harvey and Recely, 2002; McIntosh, 2003) but recently several methods have been proposed to detect coronal holes automatically, which vary from simple brightness thresholding at one single wavelength (see, e.g., Abramenko, Yurchyshyn, and Watanabe, 2009; Obridko et al., 2009) to more sophisticated approaches, which attempt to identify coronal holes objectively and to separate them from other dark regions on the Sun (see, e.g., de Toma and Arge, 2005; Henney and Harvey, 2005; Scholl and Habbal, 2008; Kirk et al., 2009; Krista and Gallagher, 2009).

In this work, we combine images taken at multiple EUV wavelengths which are available from SOHO/EIT or the two STEREO/EUVIs with magnetograms and one chromospheric line to detect coronal holes. The technique is described in detail in the Appendix and is a modified version of what originally used in de Toma and Arge (2005) and in de Toma, Arge, and Riley (2005). As in our previous work, we use EUV images assembled in the form of synoptic maps to detect coronal holes. Examples of coronal hole maps are given in Figures 3-6. The approach used here was optimized to identify the area of coronal holes and at the same time, to minimize possible problems in the calibration of EUV images. In our original method, we used fixed intensity thresholds to separate coronal holes from quiet Sun regions. This requires a good relative calibration for EUV images, so they have the same relative intensity scale. Because of the strong degradation of EUV detectors, such relative calibration is hard to achieve over the course of a long space mission like SOHO. We, thus, decided to derive the intensity thresholds used to identify coronal holes for each synoptic map, as described in the Appendix.

\subsection{Coronal Holes During the 1996 Minimum}

During the 1996 solar minimum, the two large polar coronal holes of opposite magnetic polarity were the dominant coronal holes on the Sun and stable sources of fast solar wind over the poles. This is a common coronal hole configuration at solar minimum which gives a relatively slow solar wind at the Earth, originating mostly from the edges of the polar coronal holes and, when present, from their low-latitude extensions. Typical coronal hole maps for the 1996 minimum are illustrated in Figure 3 for CR 1907 and CR 1911, in March April and July - August 1996, respectively. EUV synoptic maps are shown at the top and the corresponding coronal hole maps at the bottom. They show large polar coronal holes which extend down below $50^{\circ}$ latitude.

Boundaries of coronal holes are continuously changing so, while polar coronal holes are very longevous, they appear slightly different in shape and size every solar rotation, even near solar minimum. To estimate the area of the polar coronal holes, we derived coronal hole maps, using EIT data as described in the Appendix, for CR 1906-1917, covering the period from February 1996 to December 1996. We find that the northern polar hole was on average $12-14 \%$ larger than the southern one. We estimate that the maximum area of the polar coronal holes in 1996 was about $7.9-8.1 \%$ of the Sun's surface for the northern polar hole and $6.9-7.1 \%$ for the southern one. These area values, as well as the shape of the polar coronal holes, are in good agreement with the ones found by Harvey and Recely (2002) using the IR He I $1083 \mathrm{~nm}$ line to detect coronal holes. Isolated, low-latitude coronal holes were largely absent in 1996 and early 1997. Their total area covered typically less than $0.5 \%$ of the Sun's surface, except for a short period in the summer of 1996, corresponding to CR 1913 - 1916, when a long and narrow extension formed from the northern polar hole 


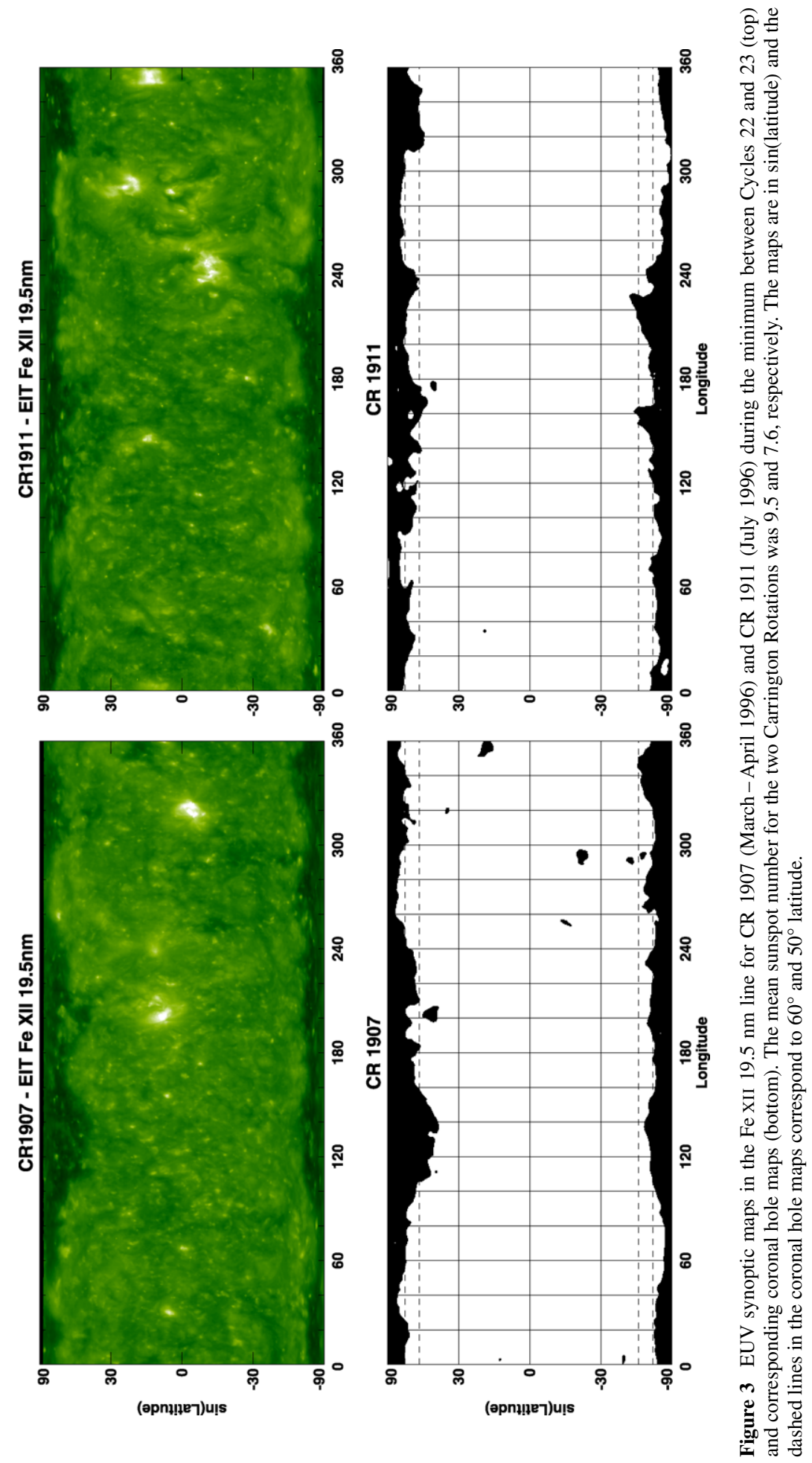



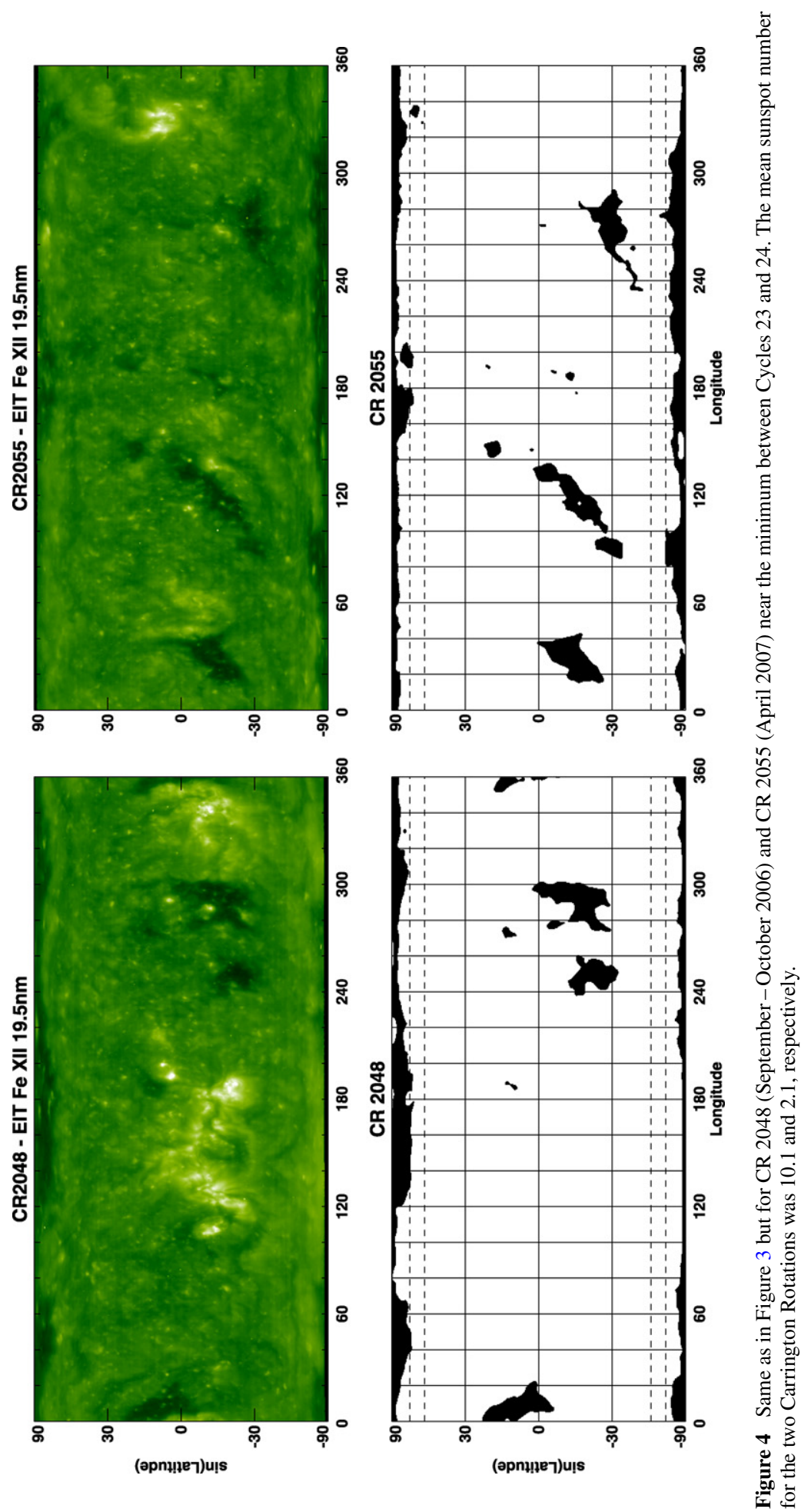


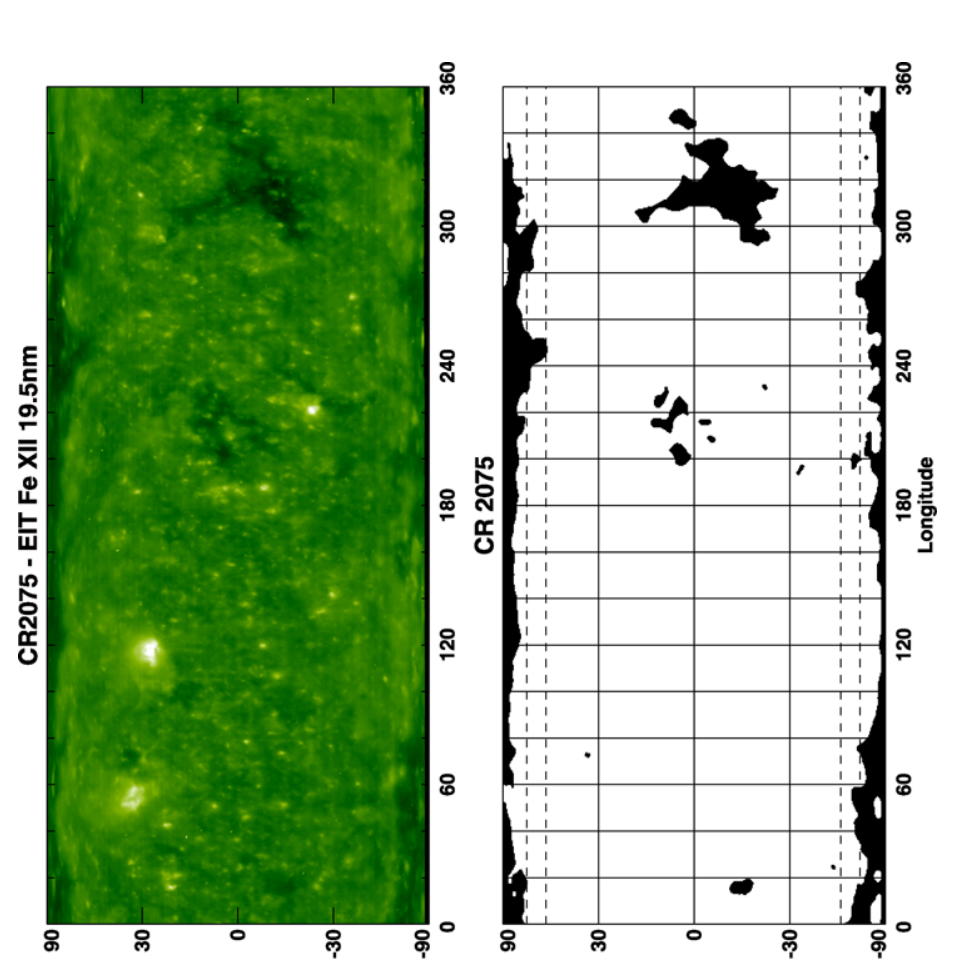

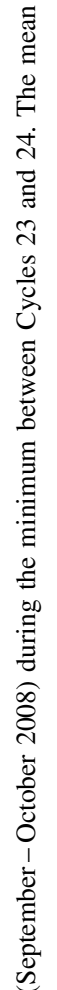
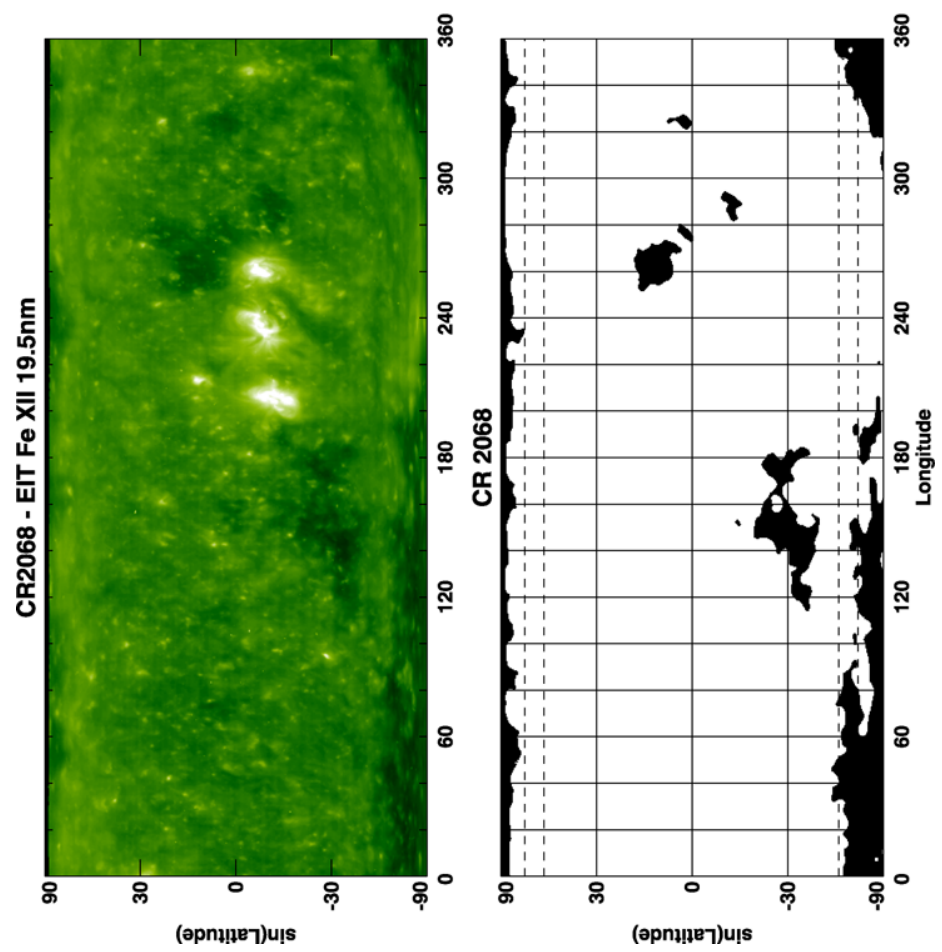

กิ 즌

के के

त.

完产

웅

들

$\sum \frac{\pi}{3}$

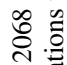

艺

흐

ㄹ.

๑

ప્心

江

.

ปิ

离

in

苋 


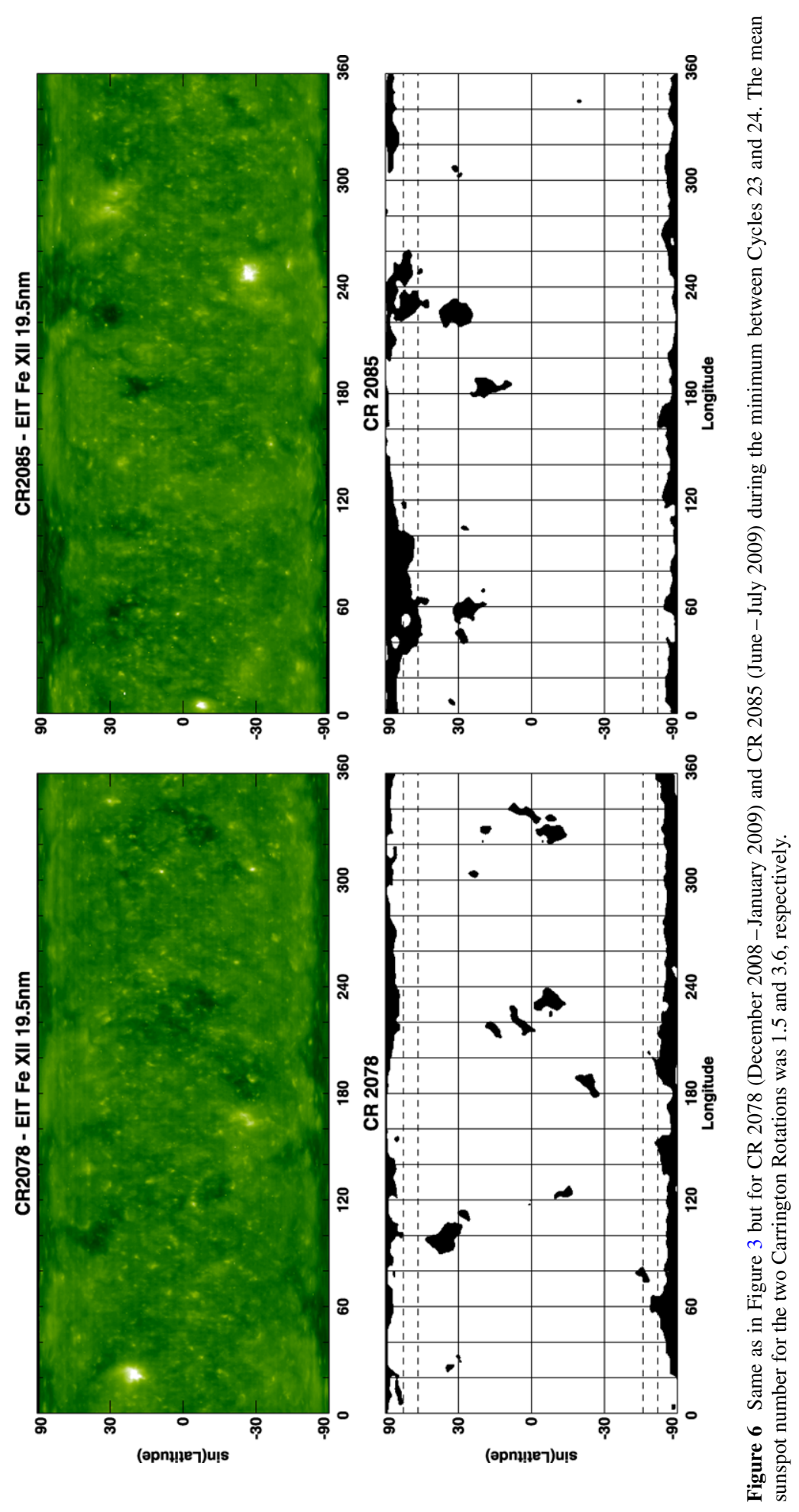


connecting it to a decayed active region located in the southern hemisphere near the equator, and later developed into detached coronal holes (Zhao, Hoeksema, and Scherrer, 1999). Even during this time, the coronal hole area at low latitudes was limited in latitudinal extent and remained below or near 1\% of the Sun's surface, except for CR 1915 for which it approached $2 \%$.

\subsection{Coronal Holes During the 2007-2009 Period}

During the long quiet period from 2007 to 2009, polar coronal holes were smaller than in 1996 , mostly confined above $60^{\circ}$ latitude, and more patchy in their appearance, as shown in Figures 4-6. This made it more difficult to identify polar coronal holes during the recent minimum. In 1996, because of the large size of the polar coronal holes, their boundaries were more easily detected, while in 2007-2009 polar coronal hole boundaries were often obscured by bright features, as can be seen in Figures $3-7$. To estimate the area occupied by coronal holes during the recent minimum, we constructed 27 coronal hole maps for EIT between September 2006 and September 2009, and 31 coronal hole maps for EUVI A and B. Figure 7 shows EUV intensity maps for EIT, EUVI B, and EUVI A at $19.5 \mathrm{~nm}$ for CR 2085, which corresponds to the period from 27 June to 24 July 2009 from the Earth's view, but which is observed at an earlier time by STEREO B and at a later time by STEREO A. The time difference with SOHO is about 3.6 days for STEREO B and about 4.0 days for STEREO A. We note that coronal holes appear partially different in the three synoptic maps, because of the temporal evolution mentioned above. Also, the STEREO satellites are close to, but not exactly on, the ecliptic plane; so the three satellites have different latitudes relative to the Sun's equator and obscuration of the poles is different in the three maps, with STEREO A giving the best view of the north pole and STEREO B of the south pole. This example clearly demonstrates that a non-favorable viewing angle can underestimate the polar coronal hole area. To remedy this effect, we weighted the He II $30.4 \mathrm{~nm}$ line more at high latitudes, as described in the Appendix, and we used only synoptic maps when one polar region was well observed, because of the favorable viewing angle to determine the coronal hole area at that pole. Another complication was the presence of more bright points within polar coronal holes in 2007-2009 than in 1996. This is possibly because magnetic flux within the polar regions was more mixed in polarity in 2007-2009 than in 1996 (de Toma and Arge, in preparation). These bright points gave rise to small "holes" within the polar coronal holes in our detection scheme. We assumed that these small bright regions were part of the coronal holes when computing the coronal hole area.

Coronal hole maps and the corresponding intensity maps are given in Figure 4 for CR 2048 and CR 2055, in the late declining phase of Cycle 23, when sunspot activity was already very low, and in Figure 5 and 6 for 2008 and 2009, during the extended minimum between Cycle 23 and Cycle 24. We find that the southern polar coronal hole was larger than the northern one and slightly increased from 2006 to 2009. The maximum area of the polar coronal holes was about $4.4-4.7 \%$ of the Sun's surface for the northern polar hole and 5.3-6.0\% for the southern one. This is a large decrease from the 1996 minimum: The northern polar hole was about 40-45\% smaller than in 1996, and the southern one about $15-25 \%$ smaller. While polar coronal holes were smaller during the recent minimum than during the 1996 minimum, low-latitude coronal holes, which are common during the declining phase, were still present throughout most of the minimum period, even when sunspot activity and magnetic emergence had dropped to levels well below those observed in 1996. These low-latitude coronal holes were prominent until the end of 2008 as shown in Figures 4 and 5. Their total area was of the order of $2-3 \%$ of the Sun's surface during 2007 

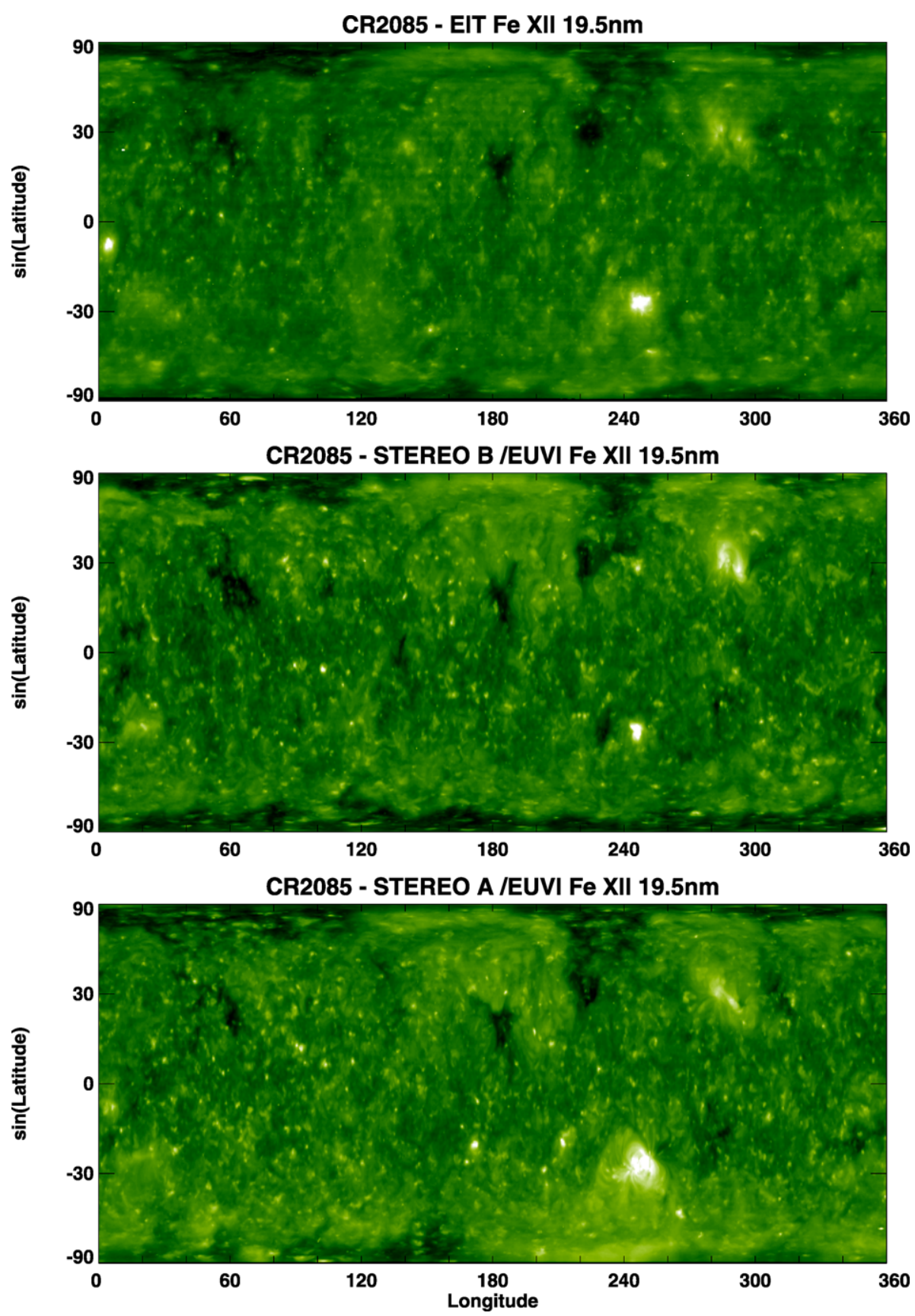

Figure 7 Example of intensity maps for EIT (top), EUVI B (middle) and EUVI A (bottom) for CR 2085 that from the Earth's view spans the period 26.6 June to 23.8 July 2009. The separation in longitude with the Earth during this period varied from $-47.5^{\circ}$ to $-49.2^{\circ}$ for STEREO B and from $+53.2^{\circ}$ to $+56.2^{\circ}$ for STEREO A. The heliographic latitude of the solar disk changed from $-3.7^{\circ}$ to $-0.8^{\circ}$ as seen from STEREO B, from $+7.0^{\circ}$ to $+7.3^{\circ}$ as seen from STEREO A, and from $+2.4^{\circ}$ to $+5.2^{\circ}$ as seen from the Earth (b-angle). 
and 2008. These relatively large and long-lived, isolated coronal holes remained important sources of the fast solar wind at the Earth during these years. By the end of 2008, we started to see the disappearance of these low-latitude, isolated coronal holes. The coronal hole map for CR 2075 in October 2008 in Figure 5 shows the last of these large, low-latitude coronal holes. By early 2009, most the low-latitude coronal holes had closed down, as shown by the coronal hole map for CR 2078 in December 2008 - January 2009 (WHI2) in Figure 6. At about the same time, with the increase of the new Cycle 24 activity, we saw the appearance of small, mid-latitude coronal holes, as illustrated in the coronal hole map for CR 2085 in July 2009 (WHI3) in Figure 6. These small coronal holes were rapidly changing and fairly transient (Wang et al., 2010). We find that their combined area was less than $1.5 \%$ of the Sun's surface. These smaller and short-lived coronal holes were not significant sources of solar wind at the Earth. Thus, the new coronal hole configuration shifted the sources of the solar wind at the Earth to higher latitudes, with the solar wind coming mostly from the edges of the polar coronal holes and occasionally from the small, mid-latitude coronal holes.

\section{Solar Wind Speed in 2007-2009}

While the interplanetary magnetic field (IMF) was lower than during other solar minima and continued to slowly decrease during the entire minimum phase reaching the lowest values ever recorded (Lee et al., 2009; Wang, Robbrecht, and Sheeley, 2009), the mean solar wind speed was not significantly different in 2007 and 2008 from that observed during other minima. Then it sharply declined to very low values in 2009 . Table 2 gives the yearly mean solar wind speed near the Earth and the magnitude of the IMF for the previous minima and for the recent years, in a format similar to the one used in Table1 for sunspots. Averages are computed using hourly data from the OMNI database (http://omniweb.gsfc.nasa.gov). The mean solar wind speed measured by the WIND and ACE satellites at 1 AU in 2006-2008 was comparable to the mean speed in 1996 as well as during the previous minima in 1986 and 1976. This is consistent with observations outside of the ecliptic plane made by Ulysses, which did not show large changes in solar wind velocity over the poles during its third orbit in 2007-2008 compared to its first orbit in 1995 near the minimum between Cycles 22 and 23 (McComas et al., 2008).

Although the mean solar wind velocity did not differ much in 2007 and 2008 from the mean velocity during other minima, there were interesting differences, as illustrated in Figure 8. In 2007 and 2008 the velocity distribution was different from 1996 and reminiscent of the distribution often seen during the declining phase of the solar cycle: It was an almost bimodal distribution with a primary peak at slightly lower velocities than in 1996 and a secondary peak near $600 \mathrm{~km} \mathrm{~s}^{-1}$. The high velocity tail in 2007 and 2008 was due to recurrent, high-speed streams originating in the large, low-latitude coronal holes present at the Sun

Table 2 Yearly averaged solar wind speed and IMF $|\mathbf{B}|$ for the solar minima in 1976, 1986, and 1996 and for the recent years. Values are computed averaging hourly data obtained from the OMNI data set. The mean velocity in 2006-2008 was similar to the velocity previously observed near solar minimum but decreased significantly in 2009. The IMF, instead, in 2006-2007 was already lower than during the recent minima and continued to slowly decline in 2008 and 2009.

\begin{tabular}{llllllll}
\hline Year & 1976 & 1986 & 1996 & 2006 & 2007 & 2008 & 2009 \\
Solar wind speed $\left(\mathrm{km} \mathrm{s}^{-1}\right)$ & 445.4 & 452.8 & 422.7 & 429.5 & 439.7 & 448.5 & 364.4 \\
$|\mathbf{B}|(\mathrm{nT})$ & 5.48 & 5.76 & 5.11 & 5.03 & 4.48 & 4.24 & 3.93 \\
\hline
\end{tabular}




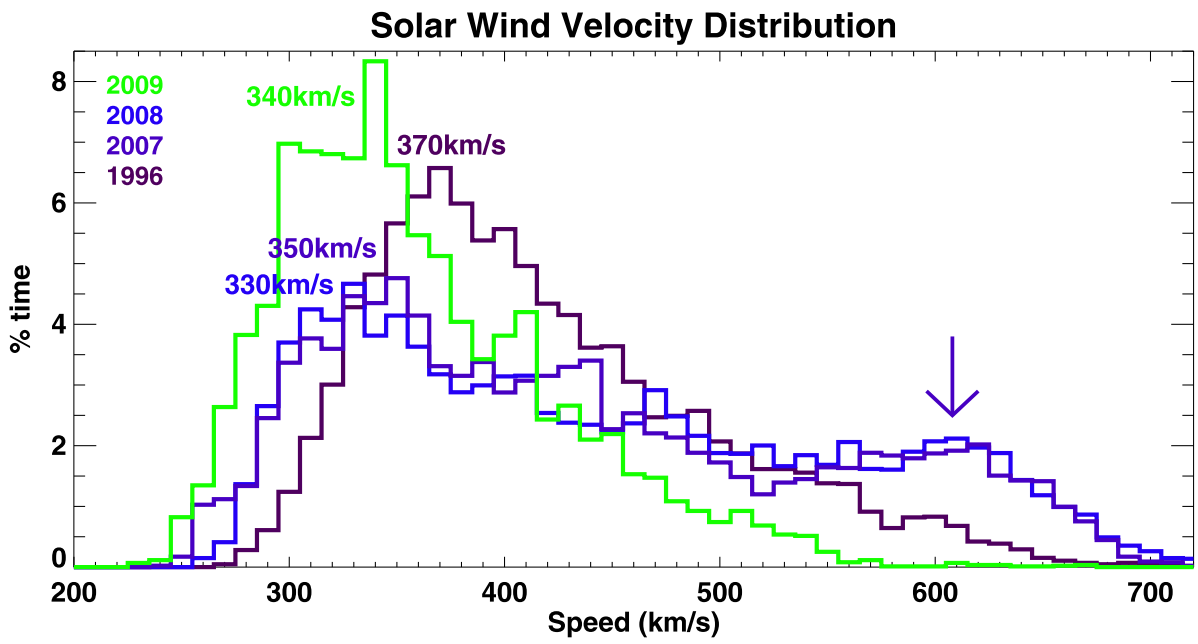

Figure 8 Histogram showing the solar wind speed distribution for 1996 (in purple), 2007 and 2008 (in blue), and 2009 (in green). The peak of the distribution is at $370 \mathrm{~km} \mathrm{~s}^{-1}, 350 \mathrm{~km} \mathrm{~s}^{-1}, 330 \mathrm{~km} \mathrm{~s}^{-1}$, and $340 \mathrm{~km} \mathrm{~s}^{-1}$, respectively. In 2007 and 2008 there is a secondary peak at about $600 \mathrm{~km} \mathrm{~s}^{-1}$.

during these years. In 2009, with the close down of these large coronal holes, the high velocity tail disappeared and velocities above $600 \mathrm{~km} \mathrm{~s}^{-1}$ became almost completely absent in the solar wind seen at the Earth. Figure 8 shows a clear shift in the distribution to the left, i.e. to lower velocities, in 2009 with speeds above $360 \mathrm{~km} \mathrm{~s}^{-1}$ systematically less frequent than in 1996. Hourly averaged solar wind speeds had values below $360 \mathrm{~km} \mathrm{~s}^{-1}$ about $57 \%$ of the time in 2009 but only $22 \%$ of the time in 1996.

Figure 9 shows the time series of the solar wind speed for the years 1996, 2007, 2008, and 2009. We see that the solar wind was faster and more structured in 2007 and 2008 than in 1996 with regular, high-speed streams. This is when the effect of the large, lowlatitude coronal holes was more evident in the solar wind near the ecliptic plane. Because these coronal holes were long-lived, the associated high-speed streams were seen for several rotations and gave rise to periodicities at 9-, 13.5- and 27-day (Emery et al., 2009; Gibson et al., 2009; Lei et al., 2010). As indicated by the hourly OMNI solar wind data, in 1996, the solar wind speed exceeded $550 \mathrm{~km} \mathrm{~s}^{-1}$ only $8 \%$ of the time compared to $21 \%$ and $23 \%$ of the time in 2007 and 2008, respectively. So, even if the mean solar wind speed did not change much, fast wind was more common in 2007-2008 than in 1996. The solar wind velocity at the Earth changed dramatically with the disappearance of the low-latitude coronal holes in late 2008. In 2009 the solar wind was not organized anymore in fast and quasiperiodic wind streams, as shown in Figure 9. The solar wind in 2009 was more similar to that observed in 1996 but with even lower velocities. The average solar wind speed dropped by about $23 \%$ from 2008 to 2009 and velocities above $550 \mathrm{~km} \mathrm{~s}^{-1}$ were observed less than $1 \%$ of the time in the hourly data.

\section{Conclusions}

We find a significant reduction in the area of the polar coronal holes during the recent minimum compared to the 1996 minimum. The northern and southern polar coronal holes at 

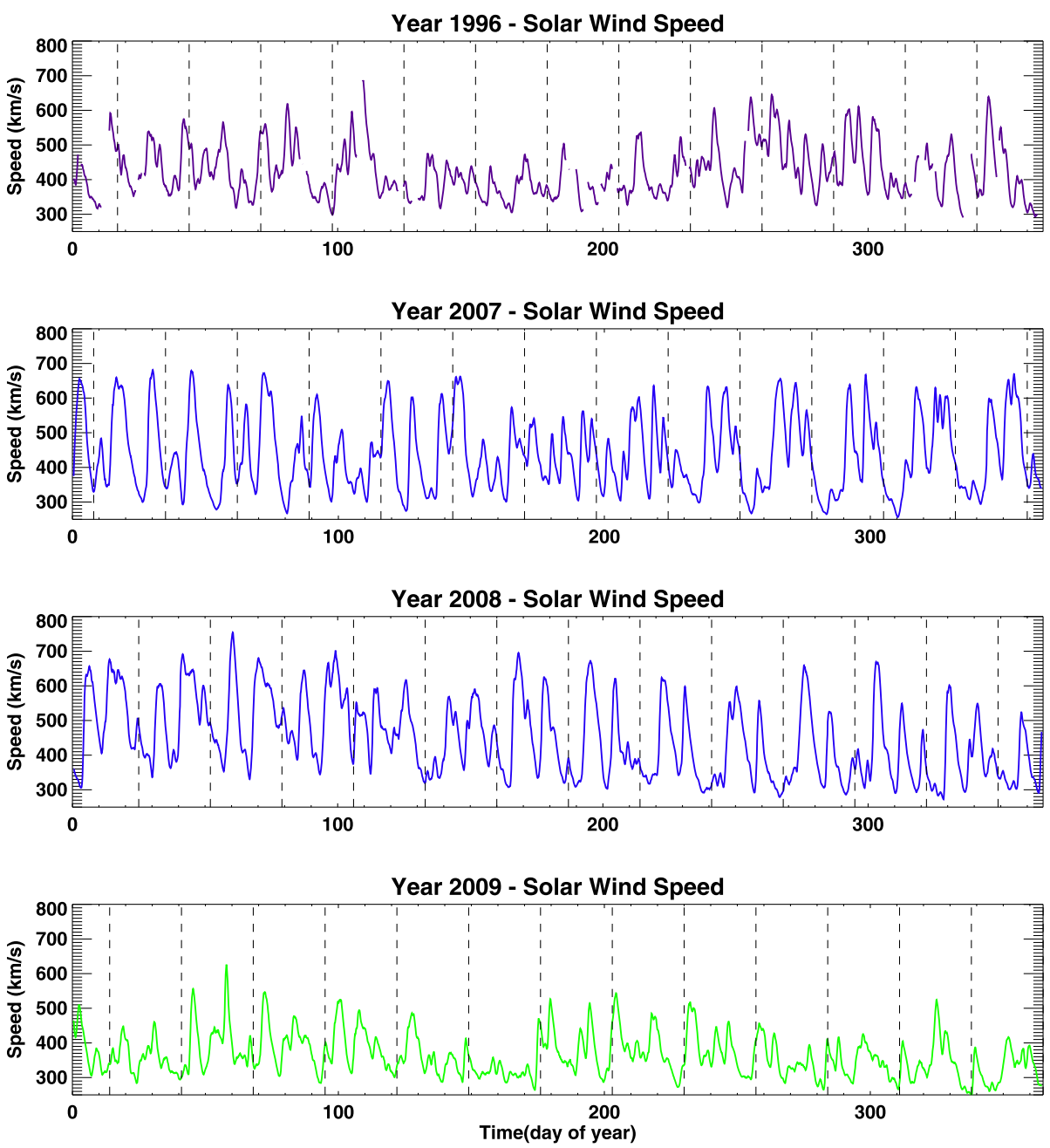

Figure 9 Time series of daily solar wind speed for the years 1996, 2007, 2008, and 2009. In 2007 and 2008 there were fast and quasiperiodic solar wind streams.

their maximum size had an area equal to about $4.4-4.7 \%$ and $5.3-6.0 \%$ of the Sun's surface, compared to $7.9-8.1 \%$ and $6.9-7.1 \%$ in 1996 , a reduction of over $40 \%$ and $20 \%$, respectively. We also find that large, low-latitude coronal holes, which were almost absent in 1996, were continuously present on the Sun and quite large in 2007 and 2008. Their total area varies from rotation to rotation with typical values between $2 \%$ and $3 \%$ of the Sun's surface. At the end of 2008, we see these large, low-latitude coronal holes closing down and the appearance of smaller, mid-latitude coronal holes that form in the remnants of the first active regions of Cycle 24. These small, mid-latitude holes occupy a smaller area, which is typically only $1-1.5 \%$ of the Sun's surface and are short-lived. This change in the distribution of coronal holes at the Sun is clearly seen in the solar wind speed at the Earth. In 2007 and 2008, the effect of multiple, large, and long-lived coronal holes resulted in regular and recurrent solar wind streams and fast wind above $550 \mathrm{~km} \mathrm{~s}^{-1}$ was still frequently observed. 
In 2009, the disappearance of the low-latitude coronal holes shifted the sources of the solar wind to higher latitudes, mostly to the edges of the polar coronal holes. This corresponds to a clear change in the solar wind speed structure, specifically to a drop of about $20 \%$ in the mean speed and the almost total disappearance of fast solar wind.

The formation of small, mid-latitude coronal holes is common when a new cycle starts and was also seen at the beginning of solar Cycle 23. When small, mid-latitude, new-cycle active regions decay they leave behind small and relatively short-lived coronal holes. However, the presence of large, low-latitude coronal holes during the minimum phase had not been previously observed in the relatively short record of coronal observations available to us. This kind of coronal holes are commonly seen during the declining phase of the solar cycle but tend to disappear during solar minimum when the polar coronal holes become dominant. The continual presence and longevity of multiple, low-latitude coronal holes during 2007 and 2008, in spite of the very low magnetic flux emergence during these years, made the minimum between Cycles 23 and 24 different from the two previous minima. The weakness of the polar magnetic field, and its associated polar dipole moment, is possibly why these isolated coronal holes were able to survive for so long.

Acknowledgements I would like to thank the WHI organizers who made this observing campaign possible, and all the WHI participants who made their data available. I am grateful to S. Gibson, C.N. Arge, J. Burkepile, and B. Emery with whom I had long discussions on the solar cycle and the impact of coronal holes on the heliosphere. This work was partially supported by the NASA LWS Grant \# NNH05AA49. The identification of coronal holes was initially developed with support from the CISM which is supported by the STC Program of the National Science Foundation under Agreement ATM-0120950. NCAR is sponsored by the National Science Foundation.

\section{Appendix: Coronal Hole Identification}

The automatic technique used to identify coronal holes uses a combination of four EUV images corresponding to the lines of Fe IX, X at $17.1 \mathrm{~nm}$, Fe XII at $19.5 \mathrm{~nm}, \mathrm{Fe}$ XV at $28.4 \mathrm{~nm}$, and $\mathrm{He}$ II at $30.4 \mathrm{~nm}$, observed by SOHO/EIT and STEREO/EUVI with magnetograms from MDI or NSO/Kitt Peak (when MDI was not available) and one chromospheric line, He I at $1083 \mathrm{~nm}$ or $\mathrm{H} \alpha$ at $656 \mathrm{~nm}$, from NSO/Kitt Peak or Mauna Loa Solar Observatory (MLSO).

Solar input data are fed to the coronal hole identification code in the form of synoptic maps. We use the standard synoptic maps for MDI, NSO/Kitt Peak, and STEREO/EUVI, which are publicly available. We assemble solar images into synoptic maps for the SOHO/EIT and MLSO data. Full-disk images are remapped into heliographic coordinates and merged in synoptic maps. We use four images per day for EIT and one image per day for $\mathrm{H} \alpha$ and eliminate images with transients, such as flares. The maps are initially constructed at a resolution close to the original resolution of the solar images with minimal averaging to avoid smearing of solar features and then re-binned to a size of $720 \times 360$ pixels. All synoptic maps used in this work are in $\sin ($ latitude), i.e. they have equal area pixels. STEREO maps are converted from latitude to $\sin$ (latitude) and reduced in size to $720 \times 360$. Their spatial resolution is also slightly reduced using a 9-pixel average to better match the resolution of the EIT images.

The first step in the code is to identify regions that are not coronal holes. For this purpose, we use magnetic maps to identify magnetic neutral lines and chromospheric maps to identify filaments. This helps us to separate coronal holes from filaments and filament channels that also appear dark at EUV wavelengths and can be confused with narrow coronal holes. This is particularly a problem in the Fe IX, X lines at $17.1 \mathrm{~nm}$ filter, where filament channels can appear as dark or darker than coronal holes. The approach used to identify magnetic inversion 
lines is similar to the one described in de Toma and Arge (2005). Magnetic maps are first smoothed, and then binary polarity maps are generated, and a Sobel filter is applied to find the boundaries between positive and negative magnetic field regions. The spatial smoothing applied depends on the instrument resolution and the original size of the map, so it is different for each instrument. We also make the assumption that coronal holes do not correspond to regions of strong photospheric magnetic fields and use magnetic maps to identify active regions as non-coronal hole regions. Filaments are identified in chromospheric maps based on their relative intensity. This information is combined in a "no holes" map.

The second step is to generate coronal hole maps for each individual EUV line. The method used here differs from the one in de Toma and Arge (2005) in two ways. It is designed to find the coronal hole area rather than the subset of the coronal holes seen at all EUV wavelengths. Secondly, the intensity thresholds used to identify coronal holes are not fixed but are determined relative to the mean quiet Sun for each Carrington map, i.e. they are allowed to vary from map to map. This approach was adopted because we found that a fixed value for the intensity thresholds did not work well for both the 1996 and 2007 2009 periods, likely because of calibration inconsistency between the early and late EIT data. EIT images were calibrated using the standard solarsoft procedure eit-prep.pro, which is designed to put EIT images on a relative intensity scale. Nonetheless, it is very difficult to fully account for instrument degradation and other instrumental effects over a period of ten years or longer. For example, starting in 2007 there is a weak horizontal band pattern in the calibrated images that appears to flip when the SOHO satellite is rolled by $180^{\circ}$ and which resembles the degradation mask applied to the data. This suggests that the pattern is a calibration artifact. Regardless of its origin, this instrumental gradient is removed before searching for coronal holes to avoid asymmetries between the two hemispheres. The intensity thresholds are then computed as follows.

First, the intensity distribution for each EUV map is computed and the peak of the histogram is assumed to correspond to the mean quiet Sun intensity, which is a reasonable assumption during solar minimum conditions. Then, the width of the distribution is derived using only the low-intensity side of the histogram to avoid contamination from active (bright) regions and assuming that the central part of the distribution is nearly Gaussian. Finally, the intensity threshold, $t h$, that separates coronal hole from the quiet Sun areas is determined as a function of the standard deviation $\sigma$, where $\sigma=\mathrm{FWHM} / 2 \sqrt{2 \ln 2}$.

The values chosen for th, or the four EIT lines at $30.4 \mathrm{~nm}, 17.1 \mathrm{~nm}, 19.5 \mathrm{~nm}$, and $28.4 \mathrm{~nm}$ are: $1.1 \times \sigma, 1.9 \times \sigma, 1.66 \times \sigma$, and $1.0 \times \sigma$; for EUVI are: $1.1 \times \sigma, 1.75 \times \sigma, 1.75 \times \sigma$, and $1.1 \times \sigma$. The values are slightly different for the two instruments, because of their different spatial resolution and relative contrast. How to choose the values for the thresholds was guided by the shape of the intensity distribution after examining all histograms. Examples of the intensity histograms for the Fe XII $19.5 \mathrm{~nm}$ line are given in Figure 10. At this particular wavelength, the histogram shows a distinct feature that appears as a small "bump" at the low end of the intensities and is well associated with coronal hole regions in solar images. The threshold $t h$ is thus chosen to match this feature. A second threshold, th2, is also chosen to identify very dark pixels that almost certainly correspond to coronal holes. This approach works quite well for the images at $17.1 \mathrm{~nm}$ and $19.5 \mathrm{~nm}$; however, images at $30.4 \mathrm{~nm}$ and $28.4 \mathrm{~nm}$ do not show such a prominent feature in the intensity histogram, especially when the total area covered by coronal holes is small. For these lines, the thresholds $t h$ and $t h 2$ were chosen by trial and error as the values that best identify coronal holes based on their relative intensity.

The intensity threshold th is used to build a binary map which is the first guess to identify coronal holes. Examples of these binary maps are given in the middle panels of Figures 11 

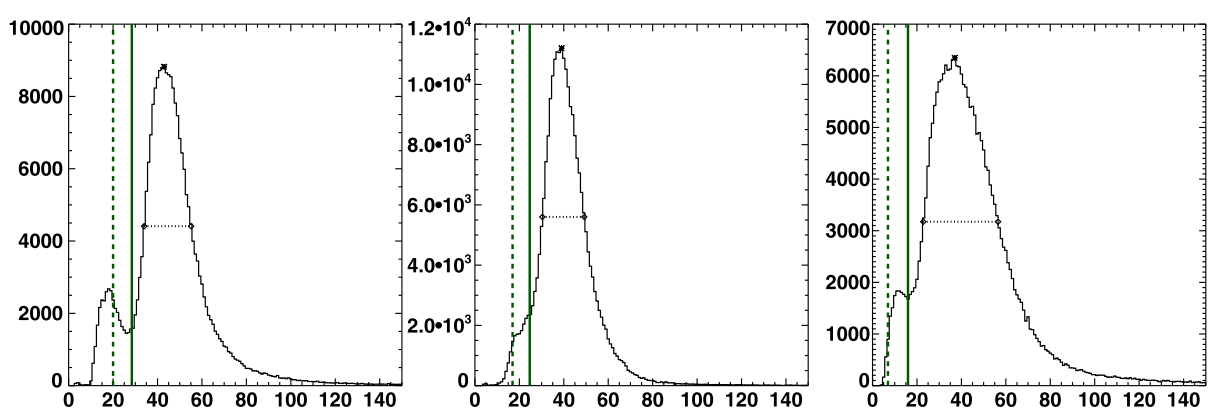

Figure 10 Example of intensity distributions for synoptic maps in the Fe XII $19.5 \mathrm{~nm}$ line for EIT (left and middle) for CR 1911 and CR 2078, and for EUVI (right) for CR 2065. The distributions show a clear feature at the low end of the intensities which is well associated with coronal holes in solar images. This corresponds to the intensity threshold th chosen to identify coronal holes, indicated by the solid vertical line. A second lower intensity th 2 , indicated by a dashed vertical line, is chosen to select very dark pixels, which almost certainly correspond to coronal hole regions.

and 12 where pixels with value below the threshold th are shown in black. Clearly, such a simple thresholding may erroneously identify small, dark, quiet Sun regions as coronal holes while small regions inside coronal holes may be not correctly identified as part of the coronal holes. Therefore, small features are filtered out and small gaps are filled in. Very dark pixels, which are in the tail of the distribution and have intensity below th 2 are added back, and pixels that were initially identified as non-coronal holes are masked out. The filling and filtering of small regions varies some from line to line, depending on the contrast of structures in that particular line. For example, the simple intensity threshold tends to identify many small features, especially at mid and low latitudes, in the He II $30.4 \mathrm{~nm}$ images that are not coronal holes. This is because the intra-network cells are very dark at this particular wavelength, so a more severe filtering is applied at these latitudes. The elimination of small features is also a function of latitude for the Fe XV $28.4 \mathrm{~nm}$ images, where the quiet Sun can be very dark during times of low activity. On the other side, bright points in the polar regions are more noticeable in the Fe lines, so an extra step is taken at these wavelengths to fill in coronal holes in the polar regions. In addition to this filtering, the ratio of the Fe IX, X $17.1 \mathrm{~nm}$ and Fe XII $19.5 \mathrm{~nm}$ also contains useful information to separate out the quiet Sun from coronal hole regions and is taken into account in building the final coronal hole map at $17.1 \mathrm{~nm}$. Examples illustrating the final coronal hole maps are given in the bottom panels of Figures 11 and 12.

The final step in the code is to combine all the individual coronal hole maps together in a weighted average, so only regions that are recognized as coronal holes at more than one wavelength are labeled as coronal holes. Because of the smaller size of polar coronal holes during the recent minimum, we found that obscuration of their lower boundary was a significant problem in the Fe lines in 2007 - 2009. To reduce this effect, the He II $30.4 \mathrm{~nm}$ line was weighted more at very high latitudes than at low latitudes. Conversely, the Fe XII $19.5 \mathrm{~nm}$ was found to give the best estimate of isolated coronal holes for EIT and was weighted more than the other lines at low latitudes. This approach gave a better identification of coronal holes during the recent minimum than using the average of the four lines, while had very little or no effect in 1996 when polar coronal holes were larger and darker; so this scheme was adopted for both minima. How maps are combined is illustrated in Figures 13, 14, and 15, which show, respectively, the four EUV intensity maps, the corresponding four coronal hole maps, and the final coronal hole map. 

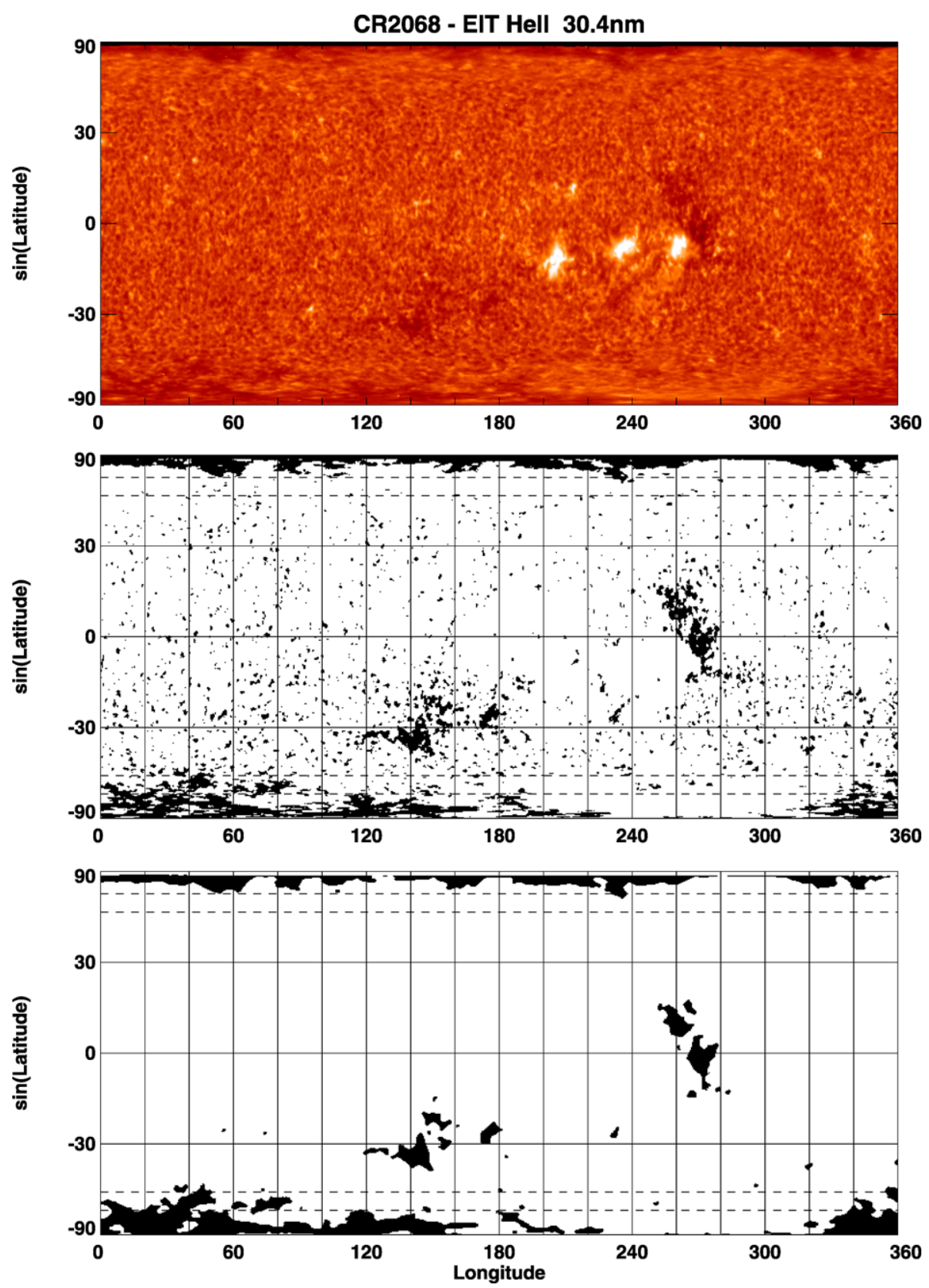

Figure 11 Example of coronal hole maps in the He II $30.4 \mathrm{~nm}$ line for the WHI CR 2068 in March-April 2008. It shows the original intensity map (top), the first mask resulting from applying a simple intensity threshold (middle), and the final map after filtering and filling (bottom).

Using multiple wavelengths gives a higher confidence that the regions found are indeed coronal holes; but clearly no simple method based on relative intensities can perfectly iden- 

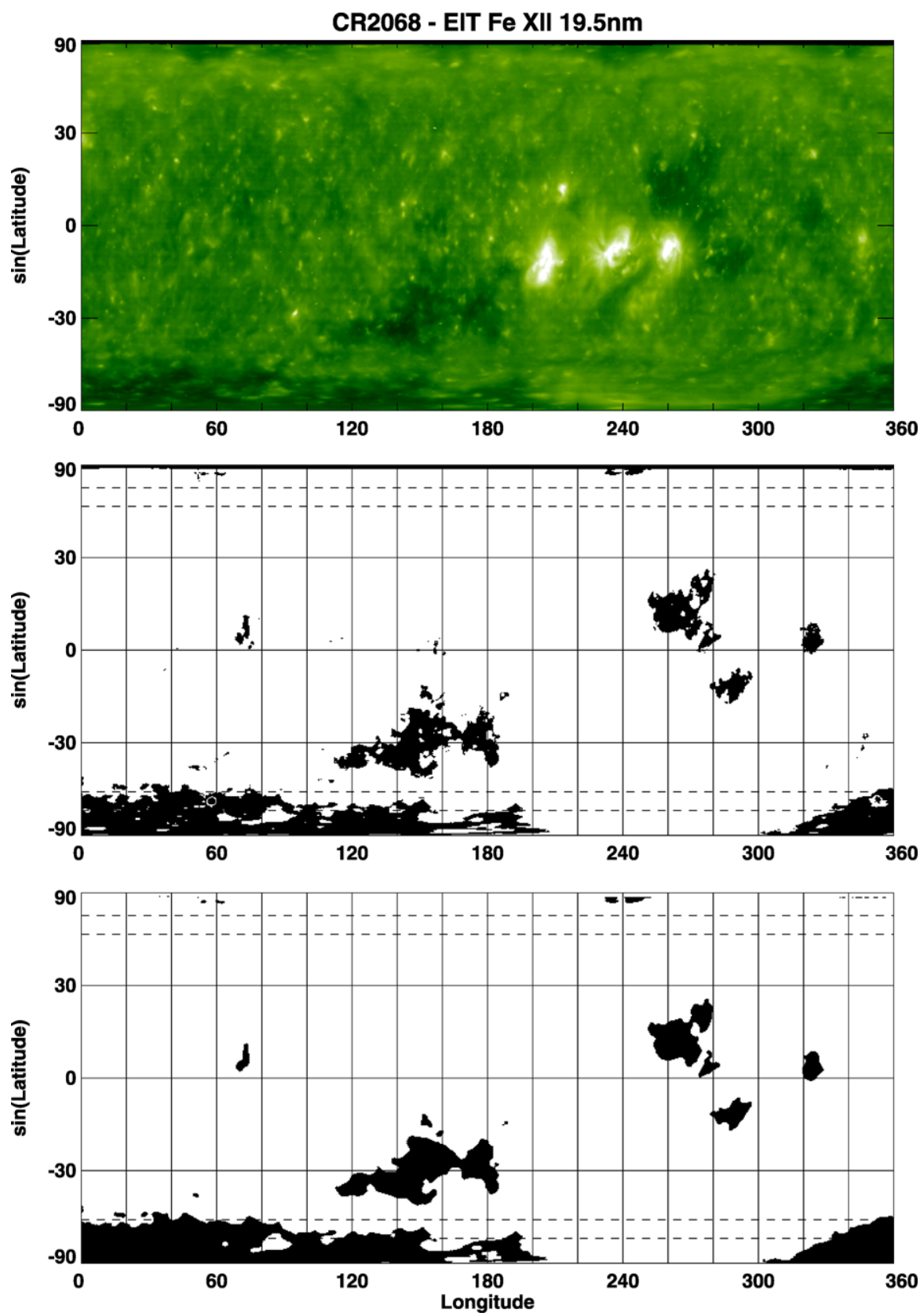

Figure 12 Example of coronal hole maps as in Figure 11 but for the Fe XII $19.5 \mathrm{~nm}$ line.

tify coronal holes. It must be kept in mind that small coronal holes may be missed or small dark regions may be erroneously labeled as coronal holes. This is true for all the intensity thresholding processes used to identify coronal holes. It is also worth noticing that estimates of the coronal hole area depend on the choice of the intensity thresholds used to identify 

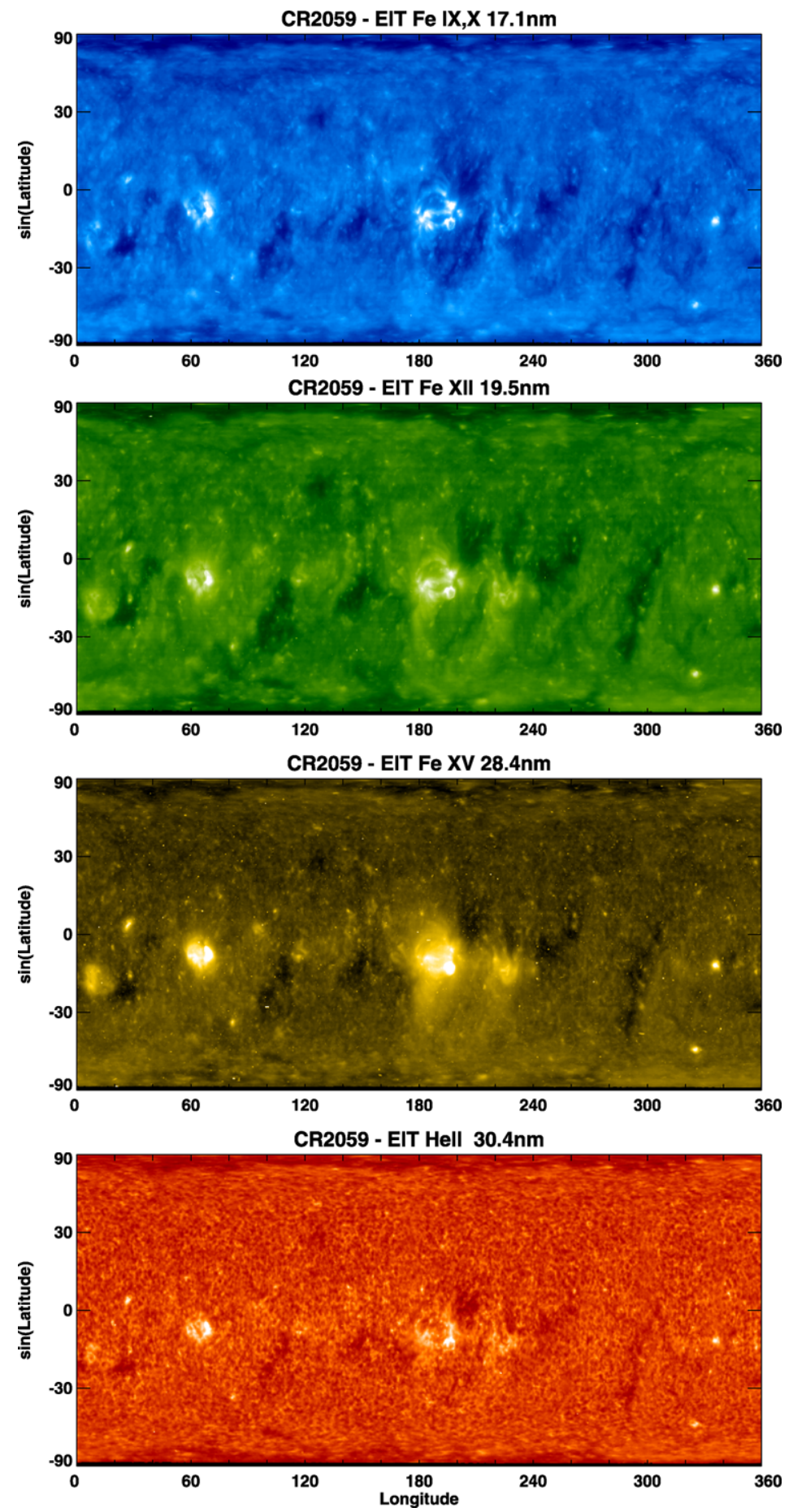

Figure 13 Intensity maps for the four EIT EUV lines at $17.1 \mathrm{~nm}$ (top), $19.5 \mathrm{~nm}$ (middle-top), $28.4 \mathrm{~nm}$ (middle-bottom), and $30.4 \mathrm{~nm}$ (bottom) for CR 2059. 

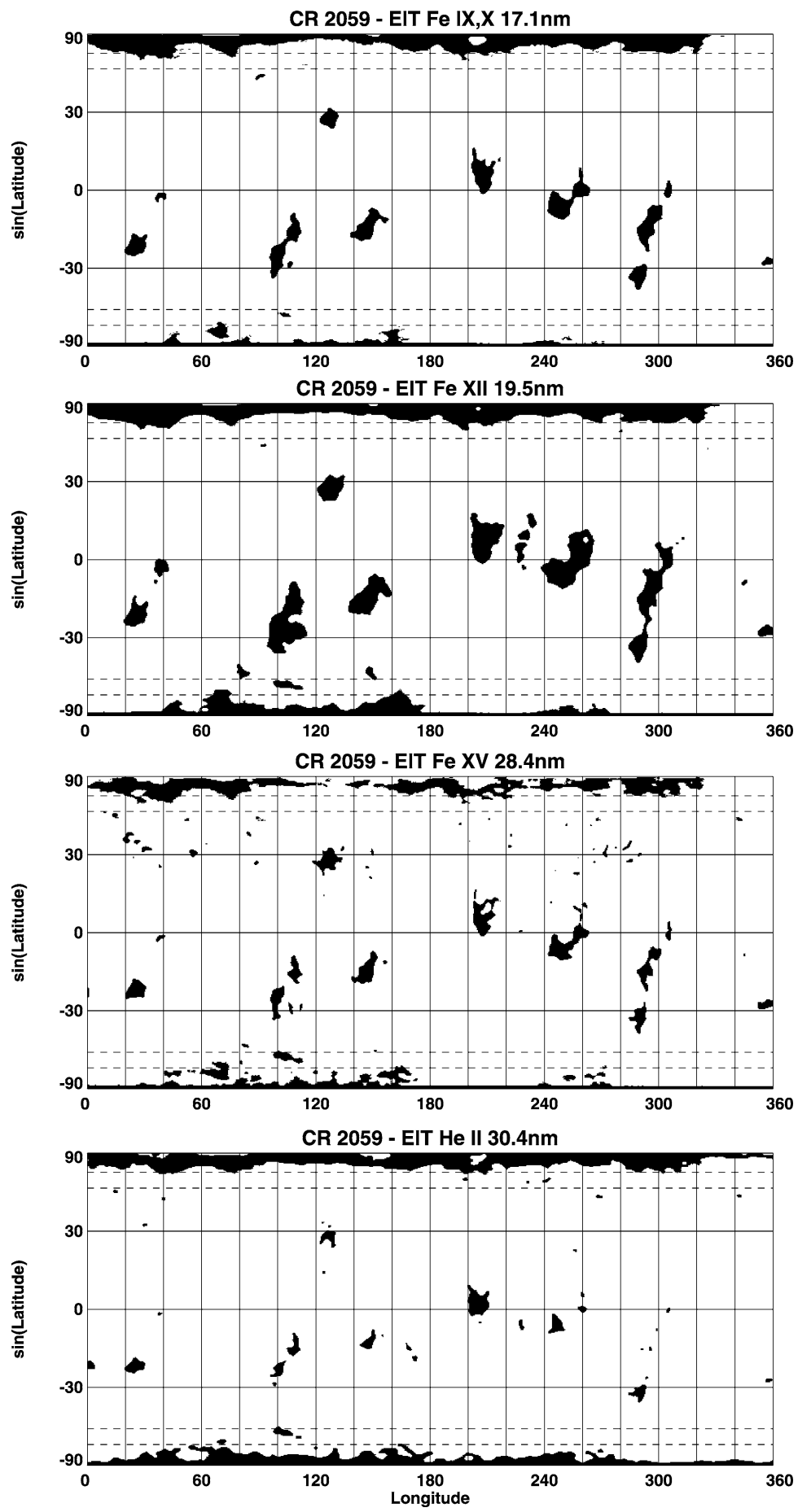

Figure 14 Coronal hole maps for the four EIT EUV lines at $17.1 \mathrm{~nm}$ (top), $19.5 \mathrm{~nm}$ (middle-top), $28.4 \mathrm{~nm}$ (middle-bottom), and $30.4 \mathrm{~nm}$ (bottom) for CR 2059. 


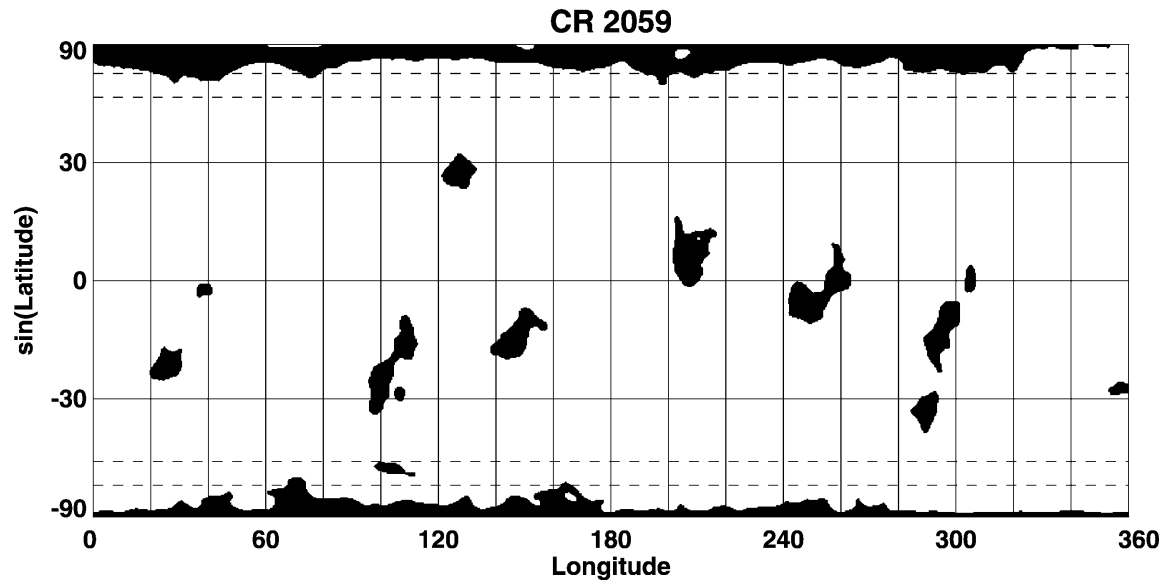

Figure 15 Final coronal hole map for CR 1959 in July - August 2007 after information from all the coronal and chromospheric lines and magnetograms is combined together.

coronal holes. We found that a change of $\pm 10 \%$ in the value of the thresholds resulted into a chance in the coronal hole area of $\pm 10-15 \%$. Larger changes in the thresholds seem to give unrealistic coronal holes, so $15 \%$ is our estimate of the uncertainty in the area associated with the choice of the intensity thresholds. In addition, there is the possible obscuration of coronal holes by bright loops. While we have taken steps to minimize this problem and used only maps with favorable b-angle, some obscuration is unavoidable and the computed coronal hole areas should be, therefore, considered to be lower limits.

\section{References}

Abramenko, V., Yurchyshyn, V., Watanabe, H.: 2009, Solar Phys. 260, 43.

Abramenko, V., Yurchyshyn, V., Linker, J., Mikić, Z., Luhmann, J., Lee, C.O.: 2010, Astrophys. J. $712,813$. Aiouaz, T., Peter, H., Lamaire, P.: 2005, Astron. Astrophys. 435, 713.

Cranmer, S.: 2009, Living Rev. Solar Phys. 6(3).

de Toma, G., Arge, C.N.: 2005, In: Penn, M., Pevtsov, A. (eds.) Large-scale Structures and their Role in Solar Activity, ASP Conf. Ser. 346, 251.

de Toma, G., Arge, C.N.: 2010, In: Maksimovic, M., et al. (eds.) Twelfth International Solar Wind Conference, AIP Conference Proceedings 1216, 679.

de Toma, G., Arge, C.N., Riley, P.: 2005, In: Fleck, B., Zurbuchen, T.H., Lacoste, H. (eds.) Solar Wind 11 /SOHO 16 Proceedings: Connecting Sun and Heliosphere SP-592, ESA, Noordwijk, 609.

de Toma, G., Gibson, S.E., Emery, B.A., Arge, C.N.: 2010a, In: Cranmer, S.R., Hoeksema, J.T., Kohl, J.L. (eds.) SOHO 23: Understanding a Peculiar Solar Minimum, ASP Conf. Ser. 428, 217.

de Toma, G., Gibson, S.E., Emery, B.A., Kozyra, J.U.: 2010b, In: Maksimovic, M., et al. (ed.) Twelfth International Solar Wind Conference, AIP Conference Proceedings 1216, 667.

Gibson, S.E., Kozyra, J.U., de Toma, G., Emery, B.A., Onsager, T., Thompson, B.J.: 2009, J. Geophys. Res. 114, A09105.

Gibson, S.E., de Toma, G., Emery, B.A., Elsworth, Y., Lei, J., McIntosh, S.W., Mewaldt, R.A., Onsager, T., Riley, P., Thompson, B.J.: 2011, Solar Phys., this issue.

Harvey, K.L., Recely, F.: 2002, Solar Phys. 211, 31.

Hassler, D.M., Dammasch, I.E., Lemaire, P., Brekke, P., Curdt, W., Mason, H.E., Vial, J., Wilhelm, K.: 1999, Science 283, 810 .

Henney, C.J., Harvey, J.W.: 2005, In: Penn, M., Pevtsov, A. (eds.) Large-scale Structures and Their Role in Solar Activity, ASP Conf. Ser. 346, 261.

Kahler, S.W., Davis, J.M., Harvey, J.W.: 1983, Solar Phys. 87, 47.

Kirk, M.S., Pesnell, W.D., Young, C.A., Hess Webber, S.A.: 2009, Solar Phys. 257, 99. 
Krista, L.D., Gallagher, P.T.: 2009, Solar Phys. 256, 87.

Krieger, A.S., Timothy, A.F., Roelof, E.C.: 1973, Solar Phys. 29, 505.

Emery, B.A., Richardson, I.G., Evans, D.S., Rich, F.I.: 2009, J. Atmos. Solar Terr. Phys. 71, 1157.

Lee, C.O., Luhmann, J.G., Zhao, X.P., Liu, Y., Riley, P., Arge, C.N., Russell, C.T., de Pater, I.: 2009, Solar Phys. 256, 345.

Lei, J., Thayer, J.P., Wang, W., McPherron, R.L.: 2010, Solar Phys., this issue.

Luhmann, J.G., Lee, C.O., Li, Y., Arge, C.N., Galvin, A.B., Simunac, K., Russell, C.T., Howard, R.A., Petrie, G.J.D.: 2009, Solar Phys. 256, 285.

McComas, D.J., Elliott, H.A., Gosling, J.T., Skoug, R.M.: 2006, Geophys. Res. Lett. 33, L09102.

McComas, D.J., Ebert, R.W., Elliott, H.A., Goldstein, B.E., Gosling, J.T., Schwadron, N.A., Skoug, R.M.: 2008, Geophys. Res. Lett. 35, L18193.

McIntosh, P.S.: 2003, In: Wilson, A. (ed.) Solar Variability as an Input to the Earth's Environment, International Solar Cycle Studies Symposium SP-535, ESA, Noordwijk, 807.

Nolte, J.T., Krieger, A.S., Timothy, A.F., Gold, R.E., Roelof, E.C., Vaiana, G., Lazarus, A.J., Sullivan, J.D., McIntosh, P.S.: 1976, Solar Phys. 46, 303.

Neupert, W.M., Pizzo, V.: 1974, J. Geophys. Res. 79, 3701.

Obridko, V.N., Shelting, B.D., Livshits, I.M., Asgarov, A.B.: 2009, Solar Phys. 260, 191.

Peter, H., Judge, P.G.: 1999, Astrophys. J. 522, 1148.

Scholl, I.F., Habbal, S.R.: 2008, Solar Phys. 248, 425.

Sheeley, N.R.: 2008, Astrophys. J. 680, 1553.

Smith, E.J., Balogh, A.: 2008, Geophys. Res. Lett. 35, L22103.

Tokumaru, M., Kojima, M., Fujiki, K., Hayashi, K.: 2009, Geophys. Res. Lett. 36, L09101.

Tokumaru, M., Kojima, M., Fujiki, K.: 2010, J. Geophys. Res. 115, A04102.

Tu, C., Zhou, C., Marsch, E., Xia, L., Zhao, L., Wang, J., Wilhelm, K.: 2005, Science 308, 519.

Xia, L.D., Marsch, E., Curdt, W.: 2003, Astron. Astrophys. 399, L5.

Wang, Y.M., Sheeley, N.R.: 2002, J. Geophys. Res. 107, 1302.

Wang, Y.M., Robbrecht, E., Sheeley, N.R.: 2009, Astrophys. J. 707, 1372.

Wang, Y.M., Robbrecht, E., Rouillard, A.P., Sheeley, N.R., Thernisien, A.F.R.: 2010, Astrophys. J. 715, 39.

Zhao, X.P., Hoeksema, J.T., Scherrer, P.H.: 1999, J. Geophys. Res. 104, 9735.

Zirker, J.B.: 1977, Coronal Holes and High-Speed Solar Wind Streams: A Monograph from Skylab Solar Workshop, Colorado Associate Univ. Press, Boulder. 